\title{
Crescimento ponderal de bezerras suplementadas com monensina sódica*
}

\section{Weight gain of heifers supplemented with monensin}

\author{
Yuri Barbosa Guerson, ${ }^{* *}$ Leonardo de Bruym Denadai, ${ }^{* * *}$ Caroline Sant'Anna Feitosa, ${ }^{* * *}$ Matheus Thomazini Oliveira, ${ }^{* * *}$ \\ Thiago Freitas da Costa, ${ }^{* * *}$ Bethânia de Souza Stein, ${ }^{* *}$ Leonardo Campos Almeida, ${ }^{* *}$ Jeanne Broch Siqueira, ${ }^{* *}$ Graziela Barioni ${ }^{* *}$
}

\section{Resumo}

O estudo teve como objetivo avaliar o efeito da inclusão de monensina sódica no concentrado inicial de bezerras lactantes, sobre o ganho de peso, perímetro torácico, altura de cernelha, altura de garupa e comprimento corporal. Foram utilizadas 12 bezerras divididas em dois grupos experimentais de acordo com a data de nascimento. Foram avaliados os tratamentos controle (sem suplementação com monensina) e suplementação diária com $0,4 \mathrm{mg}$ de monensina/ $\mathrm{kg}$ de peso corporal, a dosagem de monensina iniciou em $0,2 \mathrm{mg} / \mathrm{kg}$ peso vivo, para adaptação dos animais, sendo os animais acompanhados a partir dos 30 dias até os 86 dias de idade. Os parâmetros avaliados semanalmente foram peso corporal, perímetro torácico, altura de cernelha, altura da garupa e comprimento corporal. Tais parâmetros não apresentaram variação em função da suplementação com monensina, o menor rendimento observado para o grupo suplementado pode ter sido decorrente da diminuição do consumo. A suplementação de monensina não apresentou efeito sobre desempenho ponderal de bezerras leiteiras em sistema de desaleitamento precoce.

Palavras-chave: ganho de peso, ionóforo, medidas corporais, promotores de crescimento.

\begin{abstract}
The objective of the present study was to evaluate the effects of the inclusion of sodium monensin on the starter concentrate of lactating heifers on weight gain, chest girth, withers height, hip height and body length. Twelve heifers were divided into two experimental groups according to date of birth: a control group without supplementation and a daily supplemented group with 0.4 $\mathrm{mg}$ of sodium monensin per $\mathrm{kg}$ of body weight. The animals were observed from thirty days of age until 86 days of age. Production parameters as body weight, chest girth, withers height, hip height and body length were weekly evaluated. These parameters did not show variation depending on supplementation with monensin, the lowest yield observed in the supplemented group may have been due to lower consumption. The supplementation of monensin had no effect on weight gain of dairy calves in early weaning system.
\end{abstract}

Keywords: ionophores, growth promoters, weight gain, body measurements.

\section{Introdução}

A criação de bezerras em propriedades produtoras de leite é considerada um obstáculo dentro do orçamento da fazenda. Desta forma, tem-se tentado técnicas que reduzam o custo da energia com melhor desempenho destes animais. Animais que se tornam ruminantes mais cedo, com o ganho de peso precoce, reduzem a idade à puberdade e melhoram o desempenho produtivo quando adulto.

Os animais reposição representam de 15 a $20 \%$ das despesas da atividade, sendo as vacas em lactação os principais gastos, porém essas permitem o reembolso imediato (Signoretti et al., 2008).

O manejo nutricional é uma das áreas mais estudadas pelos pesquisadores na pecuária nacional, sendo este um fator determinante por representar a maior parte dos custos, associado à melhoria nas condições de criação para que os animais possam atingir o principal objetivo da produção expressando todo seu potencial (Van Cleef et al., 2009).
Segundo Nussio (2004), durante a fase de aleitamento o investimento com o fornecimento de leite representa $70 \%$ do custo total da criação. Para minimizar gastos na criação de bezerros, é indicado o sistema de desaleitamento precoce; entretanto, esse não pode causar nenhum prejuízo ao desempenho dos animais. Portanto, é fundamental o desenvolvimento do rúmen precocemente, fato esse associado ao consumo de matéria seca e a produção de ácidos graxos de cadeia curta permitindo a manutenção do desempenho do bezerro (Ferreira, 2007).

Alternativas para a nutrição de bezerros têm sido propostas para minimizar gastos, entre elas o fornecimento de substitutos do leite, colostro excedente, leite de descarte (Otterby e Linn, 1981) ou silagem de colostro. No entanto, os resultados desses alimentos sobre o desempenho dos bezerros são inconsistentes.

A busca por alimentos alternativos está associada a técnicas para antecipar o desenvolvimento do rúmen e o desmame prematuro, sem acarretar prejuízos ao desempenho do animal. Portanto, aditivos que proporcionam maiores concentrações dos

\footnotetext{
*Recebido em 9 de agosto de 2014 e aceito em 6 de outubro de 2015.

**Programa de Pós-Graduação em Ciências Veterinárias, Centro de Ciências Agrárias, Universidade Federal do Espírito Santo - CCAUFES/ Brasil

${ }^{* * *}$ Curso de Medicina Veterinária, Centro de Ciências Agrárias, Universidade Federal do Espírito Santo - Brasil

Autor para correspondência: yuriguerson@hotmail.com
} 
ácidos propiônico e butírico se destacam como estratégia para o desenvolvimento do rúmen, dentre eles os ionóforos.

A monensina sódica é um poliéster carboxílico utilizado na alimentação de ruminantes. Apresenta a capacidade de modificar o transporte de íons através da parede celular bacteriana, e apresenta inibição seletiva sobre as bactérias Gram-positivas. A mudança resultante nas populações de bactérias no rúmen é a alteração no padrão fermentativo (Duffield, 2008). É o ionóforo mais utilizado como aditivo alimentar e tem o papel de melhorar o metabolismo proteico e energético do animal, aumentando assim o desempenho individual e do rebanho (McGuffey; Richardson e Wilkinson, 2001).

O objetivo do trabalho foi avaliar o efeito da inclusão de monensina sódica na dieta de bezerras lactentes sobre o desempenho produtivo, acompanhando o ganho de peso, perímetro torácico, altura de garupa, altura de cernelha e comprimento corporal.

\section{Material e métodos}

O experimento foi realizado em estábulo leiteiro localizado no distrito de Anutiba, município de Alegre, Espírito Santo. Foram utilizadas 12 bezerras mestiças (Gir x Holandês) submetidas ao regime de desmame antecipado. Após o nascimento, a bezerra permaneceu com mãe nas primeiras 24 horas para a ingestão do colostro. Após a separação, os animais eram mantidos em sistema de casinha, recebendo quatro litros de sucedâneo (Nattimilk® - Auster nutrição animal) por dia, com acesso ad libitum à cana picada e ao sal proteinado. Foram fornecidos em média $300 \mathrm{~g}$ de concentrado por animal (milho, soja e trigo) diariamente, ao qual era adicionada a monensina sódica.

As bezerras foram divididas em dois grupos experimentais aleatórios, de acordo com a data de nascimento, sendo os animais nascidos em datas próximas separados um em cada grupo, para maior homogeneidade destes grupos: Grupo 1Grupo controle $(n=6)$, sem suplementação com monensina sódica e Grupo 2 - Grupo suplementado $(n=6)$ com monensina sódica a partir dos 15 dias de vida na dose diária de $0,2 \mathrm{mg} /$ $\mathrm{kg}$ de peso vivo, para adaptação durante uma semana e posteriormente manteve-se a dose diária de $0,4 \mathrm{mg} / \mathrm{kg}$ de peso vivo, realizando o ajuste semanalmente de acordo com o ganho de peso individual.

A monensina sódica foi adicionada à ração, diariamente, por um período médio de 71 dias, com adaptação anterior de uma semana utilizando a metade da dose $(0,2 \mathrm{mg} / \mathrm{kg}$ de peso vivo) estabelecida no experimento. A dose administrada era misturada a uma menor quantidade do concentrado para que não houvesse sobras do produto.

Foram realizadas as mensurações do desempenho produtivo, semanalmente aos M1 (30 dias), M2 (37 dias), M3 (44 dias), M4 (51 dias), M5 (58 dias), M6 (65 dias), M7 (72 dias), M8 (79 dias) e M9 (86 dias), por meio dos seguintes parâmetros: peso corporal, perímetro torácico, altura de cernelha, altura de garupa e comprimento corporal. A primeira mensuração dos parâmetros ponderais foi realizada após o período de adaptação a monensina realizado a partir dos 15 dias de vida.

Para tomada das medições com os animais em estação forçada, isto é, membros anterior e posterior, na perpendicular sobre um piso plano, formando um paralelogramo retangular, de forma que, visto de perfil, seus membros, para cada bípede, se encobrem e vistos de frente ou de trás estão na vertical e igualmente apoiados no piso (Hoffman, 1997)

A determinação do peso corporal foi realizada por meio da utilização da fita de pesagem, a qual foi realizada por diferentes avaliadores. A mensuração do perímetro torácico e comprimento corporal foi realizada com auxílio de uma fita flexível, com escala em centímetros e as alturas de cernelha e garupa com auxílio de medidor em aparelho que contém escala em centímetros.

Para estatística foi utilizado o programa Excel 2010 para obtenção da análise estatística descritiva (média e desvio padrão) A análise de variância (ANOVA) foi realizada por meio do programa Biostat e o teste de comparação de médias entre os grupos foi feita por meio do teste de Tukey ao nível de significância de $5 \%$.

O projeto de pesquisa foi aprovado pela Comissão de Ética no Uso de Animais (CEUA-UFES) sob protocolo n 043/2012.

\section{Resultados e discussão}

Analisando a Tabela 1, foi constatado que os animais do grupo controle apresentaram os valores médios de peso corporal e perímetro torácico superiores ao grupo suplementado no momento inicial. Essa diferença ocorreu ao acaso, pois a divisão dos grupos foi inteiramente casualizada. Os animais foram separados de acordo com a data de parição, com a finalidade de diminuir efeitos de outras variáveis como tempo, condições climáticas, estresse de manejo e oferta alimentar (capim e concentrado) durante o período, obtendo animais mais homogêneos.

A partir do momento 5 (58 dias) não se constatou mais diferença estatística entre os momentos. O menor rendimento observado nos momentos 1 (30 dias) e 4 ( 51 dias) pelo grupo suplementado em relação ao ganho de peso e no momento 1 (30 dias) para o perímetro torácico pode ser decorrente da suplementação ter ocasionado diminuição do consumo dos animais, fato relatado também por Nussio at al. (2002), Nussio et al. (2003), Klotz e Heitmann (2006) e Ferreira (2007). A redução no ganho de peso por diminuição na ingestão de alimentos foi relacionada com o aumento das doses de monensina como relatado por Goodrich et al. (1984). De acordo com Tayarol (2002), para melhorar a taxa de ingestão podem ser adicionados palatabilizantes.

Segundo Oliveira et al. (2009), a altura de cernelha isoladamente não indica se o crescimento corporal e a maturação fisiológica estão sendo adequados, devendo ser utilizadas com outros parâmetros zootécnicos, que auxiliam na avaliação do desenvolvimento desses animais. Neste caso, a razão entre o ganho de peso e a altura de cernelha foi numericamente semelhante entre os grupos, revelando que o aumento no peso foi acompanhado do crescimento e do desenvolvimento esquelético em ambos.

O ganho de peso médio total durante o ensaio foi $30,97 \mathrm{~kg}$ no controle e $27,83 \mathrm{~kg}$ para o grupo suplementado.

No entanto, os resultados encontrados para o ganho de peso divergem dos achados de Tonissi e Goes (2004) que constataram aumento no ganho de peso sem alterar o consumo alimentar com a utilização de monensina na dieta de novilhas mantidas em pastagens de média ou baixa qualidade. 
Tabela 1: Médias e desvio padrão das variáveis peso corporal $(\mathrm{Kg})$, perímetro torácico $(\mathrm{cm})$, altura de cernelha (cm), altura de garupa $(\mathrm{cm})$ e comprimento corporal $(\mathrm{cm})$ dos grupos controle (C) e suplementado (S), nos momentos: M1 aos 30 dias, M2 aos 37 dias, M3 aos 44 dias, M4 aos 51 dias, M5 aos 58 dias, M6 aos 65 dias, M7 aos 72 dias, M8 aos 79 dias, M9 aos 86 dias de idade

\begin{tabular}{|c|c|c|c|c|c|c|c|c|c|c|}
\hline & GIM & M1 & M2 & M3 & M4 & M5 & M6 & M7 & M8 & M9 \\
\hline \multirow[t]{2}{*}{ peso corporal (Kg) } & C & $46,83 \pm 2,93^{a}$ & $47,83 \pm 2,64^{a}$ & $52,17 \pm 5,64^{a}$ & $57,33 \pm 5,89^{a}$ & $59,33 \pm 6,02^{\mathrm{a}}$ & $64,67 \pm 6,06^{a}$ & $67,50 \pm 6,98^{a}$ & $72,17 \pm 11,99^{a}$ & $77,80 \pm 7,40^{\mathrm{a}}$ \\
\hline & s & $41,17 \pm 1,94^{b}$ & $45,17 \pm 2,93^{a}$ & $46,83 \pm 2,48^{a}$ & $49,33 \pm 4,72^{b}$ & $52,00 \pm 4,00^{\mathrm{a}}$ & $56,67 \pm 4,97^{a}$ & $60,67 \pm 5,54^{a}$ & $67,17 \pm 10,03^{a}$ & $69,00 \pm 16,17^{a}$ \\
\hline \multirow{2}{*}{$\begin{array}{l}\text { perímetro torácico } \\
(\mathrm{cm})\end{array}$} & C & $81,00 \pm 2,00^{\mathrm{a}}$ & $82,60 \pm 2,07^{a}$ & $84,00 \pm 3,10^{a}$ & $85,20 \pm 5,50^{\mathrm{a}}$ & $87,00 \pm 2,00^{\mathrm{a}}$ & $89,50 \pm 1,64^{a}$ & $91,50 \pm 2,88^{a}$ & $93,17 \pm 4,75^{\mathrm{a}}$ & $95,60 \pm 2,79^{a}$ \\
\hline & $s$ & $76,67 \pm 1,75^{\mathrm{b}}$ & $79,83 \pm 2,14^{a}$ & $81,60 \pm 1,14^{a}$ & $82,25 \pm 3,86^{\mathrm{a}}$ & $84,17 \pm 2,40^{\mathrm{a}}$ & $86,60 \pm 2,70^{a}$ & $88,80 \pm 2,59^{a}$ & $89,80 \pm 1,79^{a}$ & $89,25 \pm 2,99^{a}$ \\
\hline \multirow{2}{*}{$\begin{array}{l}\text { altura de cernelha } \\
\qquad(\mathrm{cm})\end{array}$} & C & $79,20 \pm 1,64^{\mathrm{a}}$ & $81,80 \pm 1,64^{a}$ & $83,17 \pm 3,13^{a}$ & $82,20 \pm 4,66^{a}$ & $82,80 \pm 2,39^{a}$ & $83,67 \pm 1,63^{a}$ & $86,33 \pm 3,83^{a}$ & $87,33 \pm 2,42^{\mathrm{a}}$ & $89,00 \pm 2,55^{\mathrm{a}}$ \\
\hline & s & $76,75 \pm 3,59^{a}$ & $77,33 \pm 3,56^{\mathrm{a}}$ & $78,50 \pm 1,73^{b}$ & $79,67 \pm 4,04^{\mathrm{a}}$ & $81,20 \pm 3,03^{a}$ & $81,00 \pm 3,39^{a}$ & $81,60 \pm 2,79^{a}$ & $83,00 \pm 3,24^{\mathrm{a}}$ & $85,25 \pm 1,71^{a}$ \\
\hline \multirow{2}{*}{$\begin{array}{l}\text { altura de garupa } \\
\qquad(\mathrm{cm})\end{array}$} & C & $83,40 \pm 1,52^{\mathrm{a}}$ & $85,60 \pm 1,52^{\mathrm{a}}$ & $86,33 \pm 3,01^{a}$ & $87,80 \pm 4,87^{a}$ & $86,80 \pm 3,56^{a}$ & $88,17 \pm 1,94^{a}$ & $88,67 \pm 1,97^{a}$ & $92,00 \pm 1,67^{a}$ & $93,20 \pm 1,79^{a}$ \\
\hline & $\mathrm{s}$ & $79,25 \pm 3,86^{a}$ & $80,17 \pm 3,54^{a}$ & $81,75 \pm 1,89^{a}$ & $82,33 \pm 3,79^{a}$ & $84,20 \pm 2,59^{a}$ & $84,80 \pm 3,90^{a}$ & $84,20 \pm 3,70^{a}$ & $85,00 \pm 2,55^{b}$ & $88,00 \pm 2,16^{b}$ \\
\hline \multirow{2}{*}{$\begin{array}{l}\text { comprimento corporal } \\
(\mathrm{cm})\end{array}$} & C & $78,00 \pm 3,39^{a}$ & $79,80 \pm 2,68^{a}$ & $81,00 \pm 3,90^{a}$ & $82,40 \pm 4,88^{a}$ & $83,00 \pm 2,74^{a}$ & $85,17 \pm 3,54^{a}$ & $87,17 \pm 3,31^{a}$ & $87,40 \pm 4,04^{\mathrm{a}}$ & $90,40 \pm 2,88^{a}$ \\
\hline & s & $76,25 \pm 3,50^{\mathrm{a}}$ & $75,50 \pm 3,62^{\mathrm{a}}$ & $78,75 \pm 2,50^{a}$ & $78,33 \pm 4,16^{a}$ & $81,20 \pm 1,92^{\mathrm{a}}$ & $84,00 \pm 2,92^{\mathrm{a}}$ & $86,40 \pm 2,30^{a}$ & $86,80 \pm 0,84^{b}$ & $86,50 \pm 2,52^{b}$ \\
\hline \multirow{2}{*}{$\begin{array}{c}\text { ganho } \mathrm{kg} \mathrm{PV} / \mathrm{cm} \text { de } \\
\text { cernelha }\end{array}$} & C & & & & & & & & & 3,16 \\
\hline & $S$ & & & & & & & & & 3,27 \\
\hline
\end{tabular}

*letras diferentes na mesma coluna indicam diferença estatisticamente significante $(p<0,05)$

${ }^{*} \mathrm{G}$ : grupos; M: momentos

O ganho de peso médio diário encontrado para o grupo controle foi $0,49 \mathrm{~kg} / \mathrm{dia}$, enquanto o grupo suplementado obteve $0,44 \mathrm{~kg} /$ dia, apresentando o grupo controle $0,05 \mathrm{~kg} / \mathrm{dia}$ de ganho de peso a mais que o grupo suplementado. Os valores encontrados para o ganho de peso diário em ambos os grupos foram semelhantes aos relatados por Rouquette Jr. et al. (1980) em novilhas.

A média de ganho de peso diário apresentou variações numéricas isoladas de acordo com o momento, em que o grupo suplementado apresentou médias superiores ao controle entre 30 (M1) e 37 (M2) dias de idade e entre os 58 (M5) e 65 (M6) dias de vida. Oliveira et al. (2005) também constataram variações entre os momentos para o ganho de peso diário sem observar influência significativa ao final do período experimental em relação ao sexo e idade. Entretanto, Salles et al. (2001) encontraram que quanto maior o nível de suplementação com ionóforo em novilhas, a taxa de ganho de peso diário pode elevar $9,8 \%$.

Aos 30, 44 e 72 dias de idade o grupo controle obteve pesos médios de 46,$83 ; 52,17$ e 67,50 kg e o grupo suplementado obteve pesos de 41,17; 46,83 e 60,67 kg, respectivamente. Os resultados encontrados nesse trabalho foram inferiores aos relatados por Heinrichs e Losinger (1998), os quais encontraram peso corporal médio em bezerros com 15 dias de idade de 53,1 kg e aos 45 e 75 dias foram de 77,2 kg e 96,1 kg, respectivamente. A alta variação entre os pesos dos bezerros entre as pesquisas também pode ser decorrente da diferença das linhagens e do melhoramento genético dos rebanhos nos Estados Unidos.

Ao comparar os valores obtidos para perímetro torácico e o peso corporal aos descritos por Oliveira e Nogueira (2006) para bezerros da raça Girolando do nascimento à desmama, as bezerras de ambos os grupos deste trabalho apresentaram desenvolvimento superior.

Por meio da medição da altura de cernelha constatou-se que o grupo controle apresentou crescimento significativamente maior no momento 3 (44 dias) para a variável altura de cernelha, com crescimento total durante o período do ensaio de $9,8 \mathrm{~cm}$ no grupo controle e de $8,5 \mathrm{~cm}$ no grupo suplementado. Já os resultados da variável altura de garupa foram significativamente maior no grupo controle nos momentos 8 e 9 (79 e 86 dias), apresentando crescimento durante o período total de $9,8 \mathrm{~cm}$ no grupo controle e $8,8 \mathrm{~cm}$ no grupo suplementado (Tabela 1 ).

Os valores de altura de cernelha aos 30 (M1), 44 (M3) e 72 (M7) dias foram: $79,2,83,17$ e $86,33 \mathrm{~cm}$ no grupo controle, e para o grupo suplementado: 76,75, 78,50 e 81, $60 \mathrm{~cm}$, respectivamente. Esses resultados corroboram com os relatados por Heinrichs e Losinger (2010) em um levantamento realizado para definir a média de tamanho de bezerros holandeses aos 15, 45 e 75 dias, os quais encontraram os valores $79,4,84,2$ e $88,0 \mathrm{~cm}$, respectivamente.

A variável das médias de comprimento corporal apresentada na Tabela 1 mostrou significância estatística com valores maiores para os animais do grupo controle aos 79 (M8) e 86 (M9) dias.

\section{Conclusões}

A inclusão de monensina sódica na dieta não melhorou 0 desempenho produtivo de bezerras lactantes, sem efeito no ganho de peso, perímetro torácico e altura de cernelha. Os animais suplementados apresentaram altura de garupa e comprimento corporal inferiores ao desmame. 


\section{Referências}

DUFFIELD, T.F. et al. A Meta-Analysis of the Impact of Monensin in Lactating Dairy Cattle. Part 2. Production Effects. Journal of Dairy Science, v. 91, n. 4, p. 1347-1360, 2008.

FERREIRA, L.S.; BITTAR, C.M.M., MATTOS, W.R.S.; ZOPOLLATTO, M.; RODRIGUES, A.A.; SANTOS, P.M.

Desempenho de bezerras leiteiras alimentadas com concentrado inicial contendo sais de ácidos orgânicos ou monensina. $44^{a}$ Reunião Anual da Sociedade e Brasileira de Zootecnia.Unesp Jaboticabal, 2007.

GOODRICH, R.D.; GARRETT, J.E.; GAST, D.R.; KIRICK, M.A.; LARSON, D.A.; MAISKE, J.C. Influence of monensin on the performance of cattle. Journal of Animal Science. v. 58, p. 14841498, 1984.

HEINRICHS, A.J.; LOSINGER, W.C. Growth of holstein dairy heifers in the united states. Journal of animal science, v. 76, n. 5 , p. $1254-1260,1998$.

HOFFMAN, P.C. Optimum body size of Holstein replacement heifers. Journal of Animal Science, v. 75, n. 3, p. 836-845, 1997.

KLOTZ, J.L.; HEITMANN, R.N. Effects of weaning and ionophore supplementation on selected blood metabolites and growth in dairy calves. Journal of Dairy Science, v. 89, n.9, p.3587-3598, 2006.

McGUFFEY, R. K.; RICHARDSON, L. F.; WILKINSON, J. I. D. Ionophores for dairy cattle: Current Status and Future Outlook. Journal of Dairy Science, v. 84, n. 1, p. 194-203, 2001.

NUSSIO, C. M. B. Custo de criação de novilhas para reposição em sistema de confinamento total. Leite DPA, Goiânia. v. 36, p. 8-11, 2004.

NUSSIO, C.M.B.; HUBER, J.T.; NUSSIO, L.G. Decoquinate, lasadocid and monensin for starter feeds and the performance of Holstein calves to 20 weeks of age. Scientia Agricola, Piracicaba, v. 59, n. 3, p. 421-426, 2002.

NUSSIO, C.M.B.; SANTOS, F.A.P.; ZOPOLLATTO, M.; PIRES, A.V.; MORAIS, L.B. Processamento de milho (floculado vs. laminado a vapor) e adição de monensina para bezerras leiteiras, pré e pós-desmama precoce. Revista Brasileira de Zootecnia. v. 32, n. 1, p. 229-239, 2003.
OLIVEIRA, D.J.C.; NOGUEIRA; G.P. Curvas de crescimento de bezerros da raça girolando. Arquivos de ciências veterinárias e zoologia da UNIPAR, v. 9, n. 1, p. 3-8, 2006.

OLIVEIRA, M.V.M.; LANA, R.P.; EIFERT, E.C., LUZ, D.F.; VARGAS, F.M.Jr. Desempenho de novilhas Holandesas confinadas com dietas com diferentes níveis de monensina sódica. Revista Brasileira de Zootecnia, v. 38, n. 9, p. 1835-1840, 2009.

OLIVEIRA, P.S.; TOMONAGA, E.H.; AMBIEL, A.C.; FERREIRA, A.C.D. Uso de uréia e ionóforos na suplementação de bezerros desmamados. Colloquium Agrariae, v. 1, n. 2, p. 28-37, 2005.

OTTERBY, D.E.; LINN, J.G. Advances in nutrition and management of calves and heifers. Journal of Dairy Science, v. 64, n. 6, p. 1365-1377, 1981.

ROUQUETTE JR., F.M.; GRIFFIN, L.R.; RANDEL, D.; CARROLL, L.H. Effect of monensin on gain and forage utilization by calves grazing bermudagrass. Journal of Animal Science, v. 51, n. 3, 1980.

SALLES, M.S.V.; ZANETTI, M.A.; CONTI, R.M.C.; LIMA, C.G. Efeitos da Monensina no Desempenho de Bezerras Leiteiras em Crescimento. Revista Brasileira de Zootecnia. v. 30, n. 4, p. 1293-1298, 2001.

SIGNORETTI, R.D.; SIQUEIRA, G.R., MIGUEL, F.B. Índices Produtivos na recria de Novilhas Leiteiras. 2008. Artigo em Hypertexto. Disponível em: http://www.infobibos.com/ Artigos/2008_2/recria/index.htm>. Acesso em: 24/9/2015.

TAYAROL, L.C. Ionóforos como Promotores de Rendimento na Pecuaria de Corte. Revista Nelore, n. 79, p. 8-12, 2002.

TONISSI, R.H.; GOES, B. Aditivos de alimento para bovinos suplementados no pasto. Cadernos técnicos de Veterinária e Zootecnia. n. 43, p. 34-45, Viçosa: UFV, 2004.

VAN CLEEF, H. E.; PATINO, P.R.; NEIVA, P.A.; SERAFIM, S.P.; REGO, C.A.; GONÇALVES, J. Distúrbios metabólicos por manejo alimentar inadequado em ruminantes: novos conceitos. Revista Colombiana Ciencia Animal. v. 1, n. 2, 2009. 